\title{
Genome-wide association study identifies genetic risk loci for adiposity in a Taiwanese population
}

\author{
Henry Sung-Ching Wong ${ }^{1}$, Szu-Yi Tsai ${ }^{1}$, Hou-Wei Chu ${ }^{2}$, Min-Rou Lin ${ }^{1}$, Gan- \\ Hong Lin $\varpi^{3}$, Yu-Ting Tai $\varpi^{4}$, Chen-Yang Shen $\varpi^{2,3,5 *}$, Wei-Chiao Chang ${ }^{1,3,6,7 *}$
}

1 Department of Clinical Pharmacy, School of Pharmacy, Taipei Medical University, Taipei, Taiwan, 2 Institute of Biomedical Sciences, Academia Sinica, Taipei, Taiwan, 3 Master Program in Clinical Genomics and Proteomics, School of Pharmacy, Taipei Medical University, Taipei, Taiwan, 4 Department of Anesthesiology, Taipei Municipal Wanfang Hospital, Taipei, Taiwan, 5 College of Public Health, China Medical University, Taichung, Taiwan, 6 Department of Pharmacy, Wan Fang Hospital, Taipei Medical University, Taipei, Taiwan, 7 Integrative Research Center for Critical Care, Wan Fang Hospital, Taipei Medical University, Taipei, Taiwan

* bmcys@ibms.sinica.edu.tw (C-YS); wcc@tmu.edu.tw (W-CC)

\section{fopen access}

Citation: Wong HS-C, Tsai S-Y, Chu H-W, Lin M-R, Lin G-H, Tai Y-T, et al. (2022) Genome-wide association study identifies genetic risk loci for adiposity in a Taiwanese population. PLOS Genet 18(1): e1009952. https://doi.org/10.1371/journal. pgen.1009952

Editor: Themistocles L. Assimes, Stanford University, UNITED STATES

Received: April 2, 2021

Accepted: November 16, 2021

Published: January 20, 2022

Copyright: ๑ 2022 Wong et al. This is an open access article distributed under the terms of the Creative Commons Attribution License, which permits unrestricted use, distribution, and reproduction in any medium, provided the original author and source are credited.

Data Availability Statement: Original individual genotype and phenotype data from the Taiwan Biobank is available for research purposes upon request and application (https://www.biobank.org. tw/about_process.php). Analyzed data including summary statistics of GWS results are available in the main text or the supplementary materials.

Funding: WCC was supported by Ministry of Science and Technology, Taiwan (MOST110-2628B-038-020 and MOST109-2628-B-038-012) and Taipei Medical University, Taiwan (12310-106079;

\section{Abstract}

Overweight and obese are risk factors for various diseases. In Taiwan, the combined prevalence of overweight and obesity has increased dramatically. Here, we conducted a genomewide association study (GWAS) on four adiposity traits, including body-mass index (BMI), body fat percentage (BF\%), waist circumference (WC), and waist-hip ratio (WHR), using the data for more than 21,000 subjects in Taiwan Biobank. Associations were evaluated between 6,546,460 single-nucleotide polymorphisms (SNPs) and adiposity traits, yielding 13 genome-wide significant (GWS) adiposity-associated trait-loci pairs. A known gene, FTO, as well as two BF\%-associated loci (GNPDA2-GABRG1 [4p12] and RNU6-2-PIAS1 [15q23]) were identified as pleiotropic effects. Moreover, RALGAPA1 was found as a specific genetic predisposing factor to high BMI in a Taiwanese population. Compared to other populations, a slightly lower heritability of the four adiposity traits was found in our cohort. Surprisingly, we uncovered the importance of neural pathways that might influence BF\%, WC and WHR in the Taiwanese (East Asian) population. Additionally, a moderate genetic correlation between the WHR and $\mathrm{BMI}\left(\mathrm{Y}_{\mathrm{g}}=0.52 ; p=2.37 \times 10^{-9}\right)$ was detected, suggesting different genetic determinants exist for abdominal adiposity and overall adiposity. In conclusion, the obesity-related genetic loci identified here provide new insights into the genetic underpinnings of adiposity in the Taiwanese population.

\section{Author summary}

Overweight and obese individuals are at risk for various medical conditions in Taiwan. Thus, we performed a genome-wide association study (GWAS) on four adiposity traits, including body-mass index (BMI), body fat percentage (BF\%), waist circumference (WC), and waist-hip ratio (WHR), using information for more than 21,000 subjects from the Taiwan Biobank. We identified in total 13 genome-wide significant (GWS) adiposity- 
Yusuke Nakamura Chair Professorship). The funders had no role in study design, data collection and analysis, decision to publish, or preparation of the manuscript.

Competing interests: The authors have declared that no competing interests exist. associated loci, which including well known genetic loci that associated with BMI, such as FTO. Additionally, we found RALGAPA1 as plausible specific susceptibility loci for BMI in Taiwanese. Surprisingly, pathway analysis revealing neuronal implication to BF\%, WC and WHR in this population.

\section{Introduction}

Obesity and overweight result from energy imbalances, which cause excessive fat accumulation in the body. Several studies reported that over the past 20 year, the prevalence of obesity has increased [1,2], and if the trends continue, obesity prevalence will reach $18 \%$ in men and $21 \%$ in women by 2025 [3]. In Taiwan, the prevalence of being overweight and obese (Body-mass index $\geq 27 \mathrm{~kg} / \mathrm{m}^{2}$ ) has grown from $11.8 \%$ to $22.0 \%$ in the past 25 years (1993 2014) [4]. Bodymass index (BMI) is a common non-invasive indicator of general adiposity, while waist circumference (WC) and the waist-hip ratio (WHR) are measurements of abdominal or visceral fat. Another index, body fat percentage (BF\%), reflects fat accumulation in adipose tissues. These measurements associate with various morbidities, such as cardiovascular diseases $[5,6]$, diabetes $[5,7,8]$, and specific types of cancers [9-11].

Family- and twin-based studies revealed that adiposity traits are highly heritable (e.g., BMI is $40 \% \sim 70 \%$ [12]; WC is $75 \%$, the WHR is $61 \%$ [13]; and $\mathrm{BF} \%$ is $64 \% \sim 74 \%[14,15]$ ); thus, genetic factors do play an important role in the development of obesity. Subsequently, largescale genome-wide association studies (GWASs) and meta-analyses have identified more than 160 loci that are associated with adiposity traits (including the BMI, BF\%, WC, WHR, obesity, body weight, and fat distribution) across different populations worldwide [16-22]. Results suggested heterogeneity across adiposity traits, revealing the differences in associated genetic loci or contributions of genetic predispositions. In European studies, BMI-associated loci were enriched in genes that implicated in the central nervous system (CNS) [16]; while WHR-associated loci were enriched in genes that involved in adipocyte metabolism. Furthermore, differences across ancestries were also observed, exemplified by significant enrichment of alcohol metabolism for BMI-associated loci in a Japanese population, but with only a weak implication of a neuronal component [23].

Hundreds of obesity susceptibility loci have been reported in GWAS meta-analyses, however, those studies mainly focused on European ancestries. Here, we conducted a GWAS for adiposity traits (including the BMI, BF\%, WC, and WHR) in a Taiwanese population. Based on the findings, we further assessed the relevant cell type, heritability, pathways implicated, and genetic correlations with adiposity. In addition, summary statistics from the UK Biobank (UKBB) GWAS, GIANT consortium, and a Japanese cohort was conducted for replication. Functional annotation was further applied to understand the biological functions of candidate adiposity genes.

\section{Results}

\section{3 significant trait-signal pairs were identified for four adiposity traits in a Taiwanese population}

In this study, data from 21,978 Taiwanese subjects in the TWB project was analyzed (Table 1). The proportions of genders were similar, and the mean age was $48.54 \pm 10.98$ years. Wholegenome imputation was conducted using a reference panel compiled by Whole genome sequence data of Taiwanese samples and the 1000 Genomes project (1KGP) EAS data, resulting in $14,555,421$ SNPs. The consistency rate of the imputation was $99.88 \% \sim 99.98 \%$ (on 
Table 1. Baseline characteristics of individuals included after genotype quality control in the genome-wide association study.

\begin{tabular}{|c|c|c|c|c|c|}
\hline Total $(N)$ & \multicolumn{5}{|c|}{21,978} \\
\hline Males $(N)$ & \multicolumn{5}{|c|}{$49.85 \%(10,956)$} \\
\hline Age (range) & \multicolumn{5}{|c|}{$48.54 \pm 10.98(30 \sim 70)$ years } \\
\hline Trait & $N$ & Range & Males (mean $\pm \mathrm{SD}$ ) & Females $($ mean \pm SD) & Total \\
\hline BMI $\left(\mathrm{kg} / \mathrm{m}^{2}\right)$ & 21,930 & $13.56 \sim 39.01$ & $25.16 \pm 3.38$ & $23.36 \pm 3.55$ & $24.26 \pm 3.58$ \\
\hline $\mathrm{BF} \%(\%)$ & 21,304 & $3.00 \sim 56.5$ & $22.89 \pm 5.46$ & $31.41 \pm 6.29$ & $27.17 \pm 7.27$ \\
\hline $\mathrm{WC}(\mathrm{cm})$ & 21,949 & $50.20 \sim 124.00$ & $87.39 \pm 9.02$ & $80.14 \pm 9.62$ & $83.75 \pm 10.0$ \\
\hline WHR & 21,972 & $0.61 \sim 1.14$ & $0.90 \pm 0.06$ & $0.84 \pm 0.07$ & $0.87 \pm 0.07$ \\
\hline
\end{tabular}

BMI, body-mass index; BF\%, body fat percentage; WC, waist circumference; WHR, waist-hip ratio; SD, standard deviation.

average for 1,283,000 SNPs). After undergoing stringent quality controls on genotypes and samples (S1 and S2 Figs), we performed a GWAS on four adiposity traits of the BMI $(n=21,930), \mathrm{BF} \%(n=21,304)$, WC $(n=21,949)$, and WHR $(n=21,972)$ using $6,546,460$ SNPs. As shown in S2 Fig, no outliers were detected from the EAS cluster in the PCA. Moreover, $\mathrm{O}-\mathrm{Q}$ plots and the genomic inflation factor $\left(\mathrm{BMI}, \lambda_{\mathrm{GC}}=1.06 ; \mathrm{BF} \%, \lambda_{\mathrm{GC}}=1.04 ; \mathrm{WC}, \lambda_{\mathrm{GC}}\right.$ $=1.04$; and WHR, $\lambda_{\mathrm{GC}}=1.03$ ) showed no inflation (S3 Fig). Therefore, further GC correction of association results was not conducted. The linkage disequilibrium (LD) score intercept of BMI, BF\%, WC and WHR were $1.0097 \pm 0.0072,1.0098 \pm 0.0067,1.018 \pm 0.0072$ and 1.0207 \pm 0.0071 , respectively.

For the four phenotypes (BMI, BF\%, WC, and WHR), we respectively detected 130, 53, 54, and 0 SNPs with genome-wide significant (GWS) association signals $\left(p<5 \times 10^{-8}\right.$; Fig 1 and S1-S4 Tables). With the exception of the WHR, genome-wide significance of the FTO locus was detected for the remaining three phenotypes. We further conducted a conditional analysis to determine the number of independent signals. By conditioning on the most significant variants of corresponding cytogenetic regions, no additional independent loci for BMI, BF\%, or WC was detected (Table 2 and S4 Fig). To annotate the functionality of adiposity trait-associated loci, we further extended the list of suggested significant SNPs with $r^{2} \geq 0.6$ and $\mathrm{MAF} \geq 0.01$. As the result, most of the variants were located in intergenic and intronic regions (85.6\% 91.4\% across the four phenotypes; S5 Fig). We further annotated the epigenetic profiles of these loci and found that over $60 \%$ of identified loci $(65.8 \% \sim 81.4 \%)$ were assigned a chromatin state across tissues. In each tissue type, nearly $30 \%$ of the loci exhibited an open state (ChromHMM state $<7$; S6 Fig), meaning that these loci likely exert biological functions by affecting the transcription.

The lead variants with $p<10^{-6}$ for each phenotype (BMI, $n=268$; BF\%, $n=100$; WC, $n=152$; and WHR, $n=25$ ) were evaluated by a meta-analysis. Additional GWAS data from the UKBB (denoted UK), GWAS catalog (denoted BBJ, GERA, and Hübel) [23,24], and GIANT consortium (denoted GIANT) was applied $[16,25]$ (S5 Table). For a given trait, metaanalyses were separately conducted for each available additional dataset using METAL software. A number of loci reached the genome-wide significance (GWS) threshold for each phenotype, including seven for BMI, two for WC and one for WHR with successful replication rates of 12\% 94\%. (S5 Table and Table 3). Accordingly, 13 unique trait-locus pairs were identified for the four adiposity traits in the Taiwanese population.

\section{Four phenotypes shared similar adiposity-associated loci}

For adiposity trait-associated loci, four (of 13;30.77\%) variants (represented by rs6567160 [BMI], rs10938397 [BMI and BF\%], rs11642015 [BF\% and WC], and rs1558902 [BMI]) 

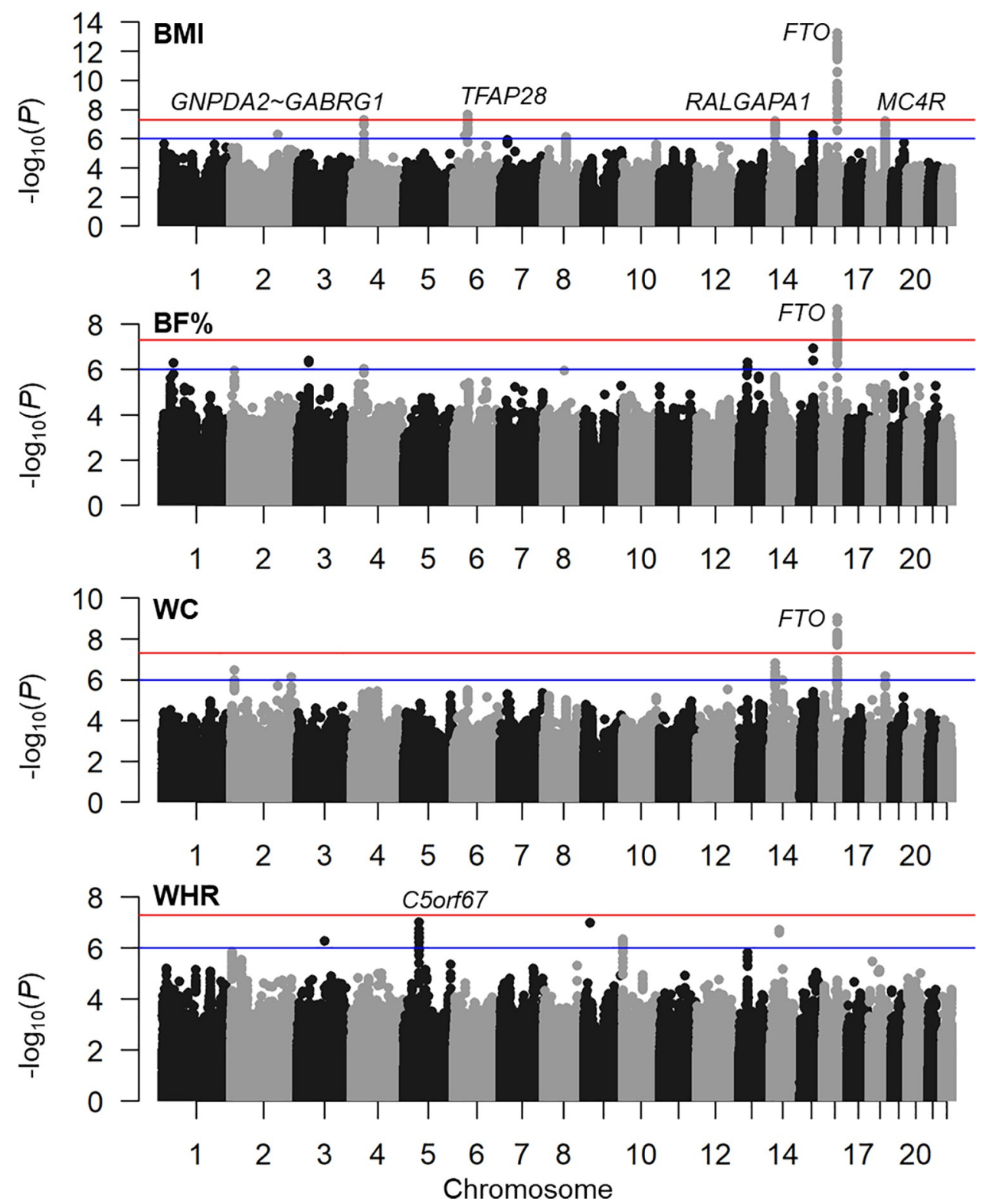

Fig 1. Manhattan plots of adiposity phenotypes. Genetic association results of the body-mass index (BMI), body fat percentage (BF\%), waist circumference (WC), and waist-hip ratio (WHR) in a Taiwanese population. Red and blue lines respectively represent genome-wide sequence significance (GWS; $p=5 \times 10^{-8}$ ) and suggested significance $\left(p=1 \times 10^{-6}\right)$.

https://doi.org/10.1371/journal.pgen.1009952.g001

exhibited pleiotropy, with between three and 19 reported associations per variant (S6 Table). Notably, all four SNPs have been reported to associate with BMI and obesity according to GWAS catalog database (extracted on November 11, 2020). In addition, most of these loci (three of four; 75\%) were identified for early-onset extreme obesity, age onset of menarche, and high-density lipoprotein (HDL) cholesterol. In this study, we also detected a locus associated with height (represented by rs6567160) [26] and two loci associated with type 2 diabetes (T2D; represented by rs11642015 and rs1558902) [23] that were consistent with results in a Japanese population. Surprisingly, we identified two unreported loci (the GNPDA2-GABRG1 locus represented by rs10938397 [chr4:45182527; cytoband 4p12; hg19] and the RNU6- 
Table 2. Independent genome-wide significant single-nucleotide polymorphisms (SNPs) identified by a conditional analysis.

\begin{tabular}{|c|c|c|c|c|c|c|c|c|c|c|}
\hline Trait & SNP & Chr. & Pos. & Alleles & MAF & $\boldsymbol{\beta}$ & SEM & $p$ value & Region & PCG(s) \\
\hline \multirow[t]{5}{*}{ BMI } & rs13130484 & 4 & 45175691 & $\mathrm{C} / \mathrm{T}$ & 0.264 & 0.059 & 0.011 & $5.15 \times 10^{-8}$ & intergenic & GNPDA2-GABRG1 \\
\hline & rs141473007 & 6 & 50855795 & $\mathrm{~A} / \mathrm{G}$ & 0.248 & -0.062 & 0.011 & $2.27 \times 10^{-8}$ & intergenic & TFAP2B-PKHD1 \\
\hline & rs8004796 & 14 & 36135219 & $\mathrm{C} / \mathrm{T}$ & 0.070 & -0.101 & 0.019 & $5.96 \times 10^{-8}$ & intronic & RALGAPA1 \\
\hline & rs1421085 & 16 & 53800954 & $\mathrm{~T} / \mathrm{C}$ & 0.126 & 0.108 & 0.014 & $5.41 \times 10^{-14}$ & intronic & FTO \\
\hline & rs72982988 & 18 & 57802714 & G/A & 0.161 & 0.072 & 0.013 & $6.28 \times 10^{-8}$ & intergenic & PMAIP1-MC4R \\
\hline $\mathrm{BF} \%$ & rs1421085 & 16 & 53800954 & $\mathrm{~T} / \mathrm{C}$ & 0.126 & 0.087 & 0.015 & $2.16 \times 10^{-9}$ & intronic & FTO \\
\hline WC & rs1421085 & 16 & 53800954 & $\mathrm{~T} / \mathrm{C}$ & 0.126 & 0.088 & 0.014 & $9.36 \times 10^{-10}$ & intronic & FTO \\
\hline
\end{tabular}

Chr., chromosome; Pos., position (based on Homo sapiens (human) genome assembly GRCh37 (hg19) from the Genome Reference Consortium); Alleles, were shown in reference/effect; MAF, minor frequency of alternative alleles; $\beta$, linear regression association coefficient (of alternative alleles); SEM, standard error of the mean; BMI, body-mass index; BF\%, body fat percentage; WC, waist circumference. PCG(s), positional candidate gene(s).

https://doi.org/10.1371/journal.pgen.1009952.t002

2-PIAS1 locus represented by rs28376697 [chr15:68140315; cytoband 15q23; hg19]) for BF\%. Since no associated signal for BF\% had previously been identified on chromosome 4 or 15 , we did not apply LD checking to the two novel loci or confirm the independence of these loci to those previously reported BF\%-associated loci. The rs10938397-A (risk) allele exhibited the higher frequencies in East Asians and Taiwanese (risk allele frequencies [RAFs] $=$ 0.697 0.731) compared to European populations (RAFs $=0.561 \sim 0.58$; based on the $1 \mathrm{KGP}$ and the genome Aggregation Database [gnomAD]; S7 Table). Similarly, the rs28376697-G (risk) allele also showed the higher frequencies in East Asians and Taiwanese population $(\mathrm{RAFs}=0.473 \sim 0.544)$ compared to European populations (RAFs $=0.188 \sim 0.226)$. Both of these $\mathrm{BF} \%$-associated variants were intergenic. We next annotated variants in $\mathrm{LD}\left(r^{2}>0.8\right.$; from the TWB) with two variants, resulting in 12 additional variants for rs10938397 and four additional variants for $\mathrm{rs} 28376697$ (S8 Table). These 18 [i.e., $(12+1)+(4+1)]$ SNPs were all in intergenic regions.

\section{Functional annotations for adiposity-associated SNPs}

Since none of these SNPs was located in an exonic region, functional annotations (FAs) of the 18 SNPs were thus inspected based on the HaploReg (v4.1) [27] and RegulomeDB [28] databases (S9 Table). Using 11 different FA criteria, we observed that one (9.09\%) to seven (63.64\%) of the 11 FAs were available for the 18 SNPs, suggesting biological relevance (through transcriptional or epigenetic regulation) of these SNPs to the susceptibility of BF\% trait. Finally, we justified that these loci were an endorsement of genetic pleiotropy of adiposity traits as follows: (1) These two loci were also associated with the BMI (based on previous GWASs and this study); (2) Both these SNPs were associated with a wide range of adiposityrelated traits based on the UK Biobank (e.g. trunk fat mass, impedance, and critically BF\% by limb).

\section{RALGAPA1 as plausible specific susceptibility loci for BMI in the Taiwanese population}

Based on our results, a GWS signal for RALGAPA1 (within cytoband 14q13.2) was identified to correlate with BMI. However, only one significant BMI signal (rs10140922) within $\pm 1-\mathrm{Mb}$ flanking region of the top signal (rs8004796) in RALGAPA1 has previously been reported [25]. In addition, the SNPs were not in LD $\left(r^{2}<0.1\right.$ in African, American, European, East Asian and South Asian based on 1000 Genomes, Phase 3 v5). We further narrowed down this region, however, no significant signal was found in either the GERA BMI [17], BBJ BMI [23], or 
Table 3. The 29 single-nucleotide polymorphisms (SNPs) that were successfully replicated (reached genome-wide sequencing significance (GWS)) by a trans-ethnic meta-analysis and corresponding 13 (significant adiposity-associated) trait-locus pairs.

\begin{tabular}{|c|c|c|c|c|c|c|c|c|c|c|c|c|c|c|}
\hline Trait & Dataset & SNP & Chr. & Pos. & $\begin{array}{l}\text { Ref/ } \\
\text { Alt }\end{array}$ & MAF & Func. region & Gene & Effect & Std.Err. & $p$ value & Dir. & $I^{2}$ & $P_{H e t}$ \\
\hline BMI & TWB+UK & rs10938397 & 4 & 45182527 & $\mathrm{~A} / \mathrm{G}$ & 0.2638 & intergenic & GNPDA2-GABRG1 & -0.0213 & 0.0018 & $3.36 \times 10^{-33}$ & - & 91.9 & 0.000447 \\
\hline BMI & TWB+UK & rs2635727 & 6 & 50820940 & $\mathrm{~T} / \mathrm{C}$ & 0.2285 & intergenic & TFAP2B-PKHD1 & -0.0191 & 0.002 & $7.06 \times 10^{-21}$ & - & 93 & 0.000157 \\
\hline BMI & TWB+UK & rs28376697 & 15 & 68140315 & $\mathrm{~A} / \mathrm{G}$ & 0.4419 & intergenic & RNU6-2-PIAS1 & 0.0178 & 0.0021 & $3.94 \times 10^{-17}$ & ++ & 89.8 & 0.001757 \\
\hline BMI & TWB+UK & rs11642015 & 16 & 53802494 & $\mathrm{~T} / \mathrm{C}$ & 0.1259 & intronic & FTO & 0.041 & 0.0018 & $1.19 \times 10^{-114}$ & ++ & 95.3 & $3.82 \times 10^{-6}$ \\
\hline BMI & TWB+UK & rs6567160 & 18 & 57829135 & $\mathrm{~T} / \mathrm{C}$ & 0.181 & intergenic & PMAIP1-MC4R & -0.0252 & 0.0021 & $5.66 \times 10^{-34}$ & - & 90 & 0.001558 \\
\hline BMI & TWB+BBJ & rs13130484 & 4 & 45175691 & $\mathrm{~T} / \mathrm{C}$ & 0.2636 & intergenic & GNPDA2-GABRG1 & 0.0315 & 0.0036 & $5.64 \times 10^{-18}$ & ++ & 86.2 & 0.007012 \\
\hline BMI & TWB+BBJ & rs57988840 & 6 & 50817748 & $\mathrm{~A} / \mathrm{T}$ & 0.2275 & intergenic & TFAP2B-PKHD1 & -0.0245 & 0.0042 & $4.65 \times 10^{-9}$ & - & 91.8 & 0.000468 \\
\hline BMI & TWB+BBJ & rs78613881 & 8 & 81403127 & $\mathrm{~T} / \mathrm{C}$ & 0.2375 & intronic & ZBTB10 & -0.0275 & 0.0044 & $2.57 \times 10^{-10}$ & - & 86 & 0.007505 \\
\hline BMI & TWB+BBJ & rs116994836 & 14 & 36127690 & $\mathrm{~T} / \mathrm{C}$ & 0.07019 & ncRNA & RALGAPA1P1 & -0.0384 & 0.0065 & $3.22 \times 10^{-9}$ & - & 91.7 & 0.000506 \\
\hline $\mathrm{BMI}$ & TWB+BBJ & rs1421085 & 16 & 53800954 & $\mathrm{~T} / \mathrm{C}$ & 0.1262 & intronic & FTO & -0.0815 & 0.0042 & $2.41 \times 10^{-83}$ & - & 73.2 & 0.05321 \\
\hline BMI & TWB+BBJ & rs6567160 & 18 & 57829135 & $\mathrm{~T} / \mathrm{C}$ & 0.181 & intergenic & PMAIP1-MC4R & -0.0524 & 0.0041 & $3.95 \times 10^{-38}$ & - & 0 & 0.3244 \\
\hline BMI & $\begin{array}{l}\text { TWB } \\
\text { +GERA }\end{array}$ & rs10938397 & 4 & 45182527 & $\mathrm{~A} / \mathrm{G}$ & 0.2638 & intergenic & GNPDA2-GABRG1 & -0.0378 & 0.0025 & $2.99 \times 10^{-53}$ & - & 74.7 & 0.04681 \\
\hline BMI & $\begin{array}{l}\text { TWB } \\
\text { +GERA }\end{array}$ & rs2817419 & 6 & 50812906 & $\mathrm{~A} / \mathrm{G}$ & 0.2318 & UTR3 & TFAP $2 B$ & 0.029 & 0.0028 & $5.70 \times 10^{-26}$ & ++ & 88.6 & 0.003094 \\
\hline BMI & $\begin{array}{l}\text { TWB } \\
\text { +GERA }\end{array}$ & rs16907771 & 8 & 81415556 & $\mathrm{~T} / \mathrm{G}$ & 0.2375 & intronic & ZBTB10 & 0.0436 & 0.0061 & $8.46 \times 10^{-13}$ & ++ & 37 & 0.2078 \\
\hline BMI & $\begin{array}{l}\text { TWB } \\
\text { +GERA }\end{array}$ & rs8004796 & 14 & 36135219 & $\mathrm{~T} / \mathrm{C}$ & 0.06993 & ncRNA & RALGAPA1P1 & -0.0807 & 0.0135 & $2.41 \times 10^{-9}$ & - & 59.8 & 0.1149 \\
\hline BMI & $\begin{array}{l}\text { TWB } \\
\text { +GERA }\end{array}$ & rs4776990 & 15 & 68137364 & $\mathrm{~T} / \mathrm{C}$ & 0.4439 & intergenic & RNU6-2-PIAS1 & -0.0283 & 0.003 & $1.23 \times 10^{-21}$ & - & 78.7 & 0.03042 \\
\hline BMI & $\begin{array}{l}\text { TWB } \\
\text { +GERA }\end{array}$ & rs1558902 & 16 & 53803574 & $\mathrm{~A} / \mathrm{T}$ & 0.1258 & intronic & FTO & 0.0798 & 0.0025 & $6.48 \times 10^{-222}$ & ++ & 72 & 0.05861 \\
\hline BMI & $\begin{array}{l}\text { TWB } \\
\text { +GERA }\end{array}$ & rs6567160 & 18 & 57829135 & $\mathrm{~T} / \mathrm{C}$ & 0.181 & intergenic & PMAIP1-MC4R & -0.0528 & 0.0029 & $3.40 \times 10^{-75}$ & - & 0 & 0.3574 \\
\hline $\mathrm{BF} \%$ & TWB+UK & rs10938397 & 4 & 45182527 & $\mathrm{~A} / \mathrm{G}$ & 0.2638 & intergenic & GNPDA2-GABRG1 & -0.0212 & 0.0018 & $1.02 \times 10^{-32}$ & - & 89.1 & 0.002493 \\
\hline $\mathrm{BF} \%$ & TWB+UK & rs28376697 & 15 & 68140315 & $\mathrm{~A} / \mathrm{G}$ & 0.4419 & intergenic & RNU6-2-PIAS1 & 0.0179 & 0.0021 & $3.14 \times 10^{-17}$ & ++ & 90.9 & 0.000907 \\
\hline $\mathrm{BF} \%$ & TWB+UK & rs11642015 & 16 & 53802494 & $\mathrm{~T} / \mathrm{C}$ & 0.1259 & intronic & FTO & 0.0406 & 0.0018 & $1.10 \times 10^{-112}$ & ++ & 89.7 & 0.001811 \\
\hline $\mathrm{BF} \%$ & TWB+Hübel & rs10938397 & 4 & 45182527 & $\mathrm{~A} / \mathrm{G}$ & 0.2638 & intergenic & GNPDA2-GABRG1 & -0.085 & 0.0098 & $3.35 \times 10^{-18}$ & - & 97.4 & $6.65 \times 10^{-10}$ \\
\hline $\mathrm{BF} \%$ & TWB+Hübel & rs28376697 & 15 & 68140315 & $\mathrm{~A} / \mathrm{G}$ & 0.4419 & intergenic & RNU6-2-PIAS1 & 0.0606 & 0.0091 & $3.32 \times 10^{-11}$ & ++ & 90.1 & 0.001512 \\
\hline $\mathrm{BF} \%$ & TWB+Hübel & rs1421085 & 16 & 53800954 & $\mathrm{~T} / \mathrm{C}$ & 0.1262 & intronic & FTO & -0.1604 & 0.0121 & $2.02 \times 10^{-40}$ & - & 98.8 & $2.46 \times 10^{-19}$ \\
\hline WC & TWB+UK & rs11642015 & 16 & 53802494 & $\mathrm{~T} / \mathrm{C}$ & 0.1259 & intronic & FTO & 0.0573 & 0.0021 & $5.26 \times 10^{-167}$ & ++ & 77.3 & 0.03597 \\
\hline WC & TWB+UK & rs9954728 & 18 & 57801557 & $\mathrm{C} / \mathrm{G}$ & 0.1802 & intergenic & PMAIP1-MC4R & -0.031 & 0.0022 & $3.88 \times 10^{-46}$ & - & 84.6 & 0.01091 \\
\hline WC & $\begin{array}{l}\text { TWB } \\
\text { +GIANT }\end{array}$ & rs9939973 & 16 & 53800568 & $\mathrm{~A} / \mathrm{G}$ & 0.1702 & intronic & FTO & 0.0725 & 0.0033 & $6.16 \times 10^{-108}$ & ++ & 0 & 0.5501 \\
\hline WC & $\begin{array}{l}\text { TWB } \\
\text { +GIANT }\end{array}$ & rs1942880 & 18 & 57793209 & $\mathrm{~T} / \mathrm{C}$ & 0.1795 & intergenic & PMAIP1-MC4R & 0.0362 & 0.0035 & $1.45 \times 10^{-25}$ & ++ & 79.2 & 0.02828 \\
\hline WHR & $\begin{array}{l}\text { TWB } \\
\text { +GIANT }\end{array}$ & rs459193 & 5 & 55806751 & $\mathrm{~A} / \mathrm{G}$ & 0.47 & downstream & C5orf67 & 0.0288 & 0.0035 & $3.11 \times 10^{-16}$ & ++ & 75.2 & 0.04455 \\
\hline
\end{tabular}

Chr., chromosome; Pos., position; Ref, reference allele; Alt, effect allele; MAF, minor frequency of alternative alleles; Effect, meta-analysis-estimated effect size; Std.Err., standard error for effect size estimate; Dir., the effect direction of each study; $I^{2}$, heterozygosity (\%); $P_{\text {Het }}$, $p$ value of the heterozygosity statistic (Chi-squared distribution with a degree of freedom of 1); BMI, body-mass index; BF\%, body fat percentage; WC, waist circumference. Gray, leading SNPs (with the highest $p$ values) of unique associated loci.

https://doi.org/10.1371/journal.pgen.1009952.t003

GWAS catalog (BMI trait) within a \pm 100 -kb region of RALGAPA1 (Fig 2). FAs revealed biological implications of RALGAPA1 for BMI, BF\%, and WC (but not WHR), which included (1) a Combined Annotation-Dependent Depletion (CADD) score of $>12.37$; (2) 15-core 


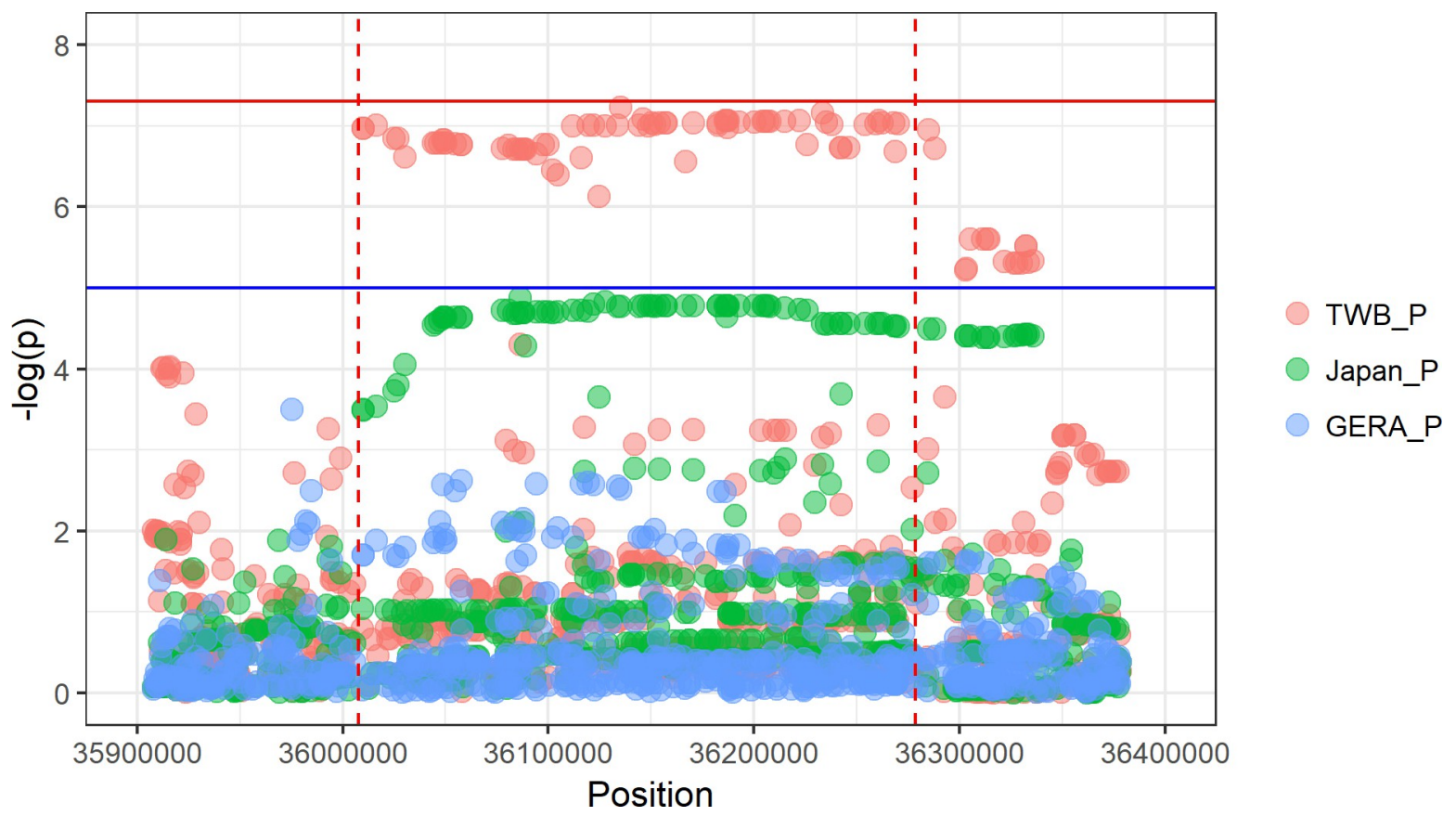

Fig 2. A novel gene, RALGAPA1, identified by comparison of Taiwan Biobank (TWB), GERA and Japan Biobank (BBJ) genome-wide association studies (GWASs). The $X$-axis represents the position of loci (hg19). The $Y$-axis represents $-\log _{10}(p)$. Red dots are $p$ values of variants from the TWB. Green dots are $p$ values from the BBJ. Blue dots are $p$ values from GERA cohort (GERA). The blue horizontal line represents $p=1 \times 10^{-5}$. The red horizontal line represents $p=5 \times 10^{-8}$. The red dashed line represents the location of the gene.

https://doi.org/10.1371/journal.pgen.1009952.g002

chromatin state mapping; and (3) 3D chromatin interaction mapping (S7 Fig). Moreover, to assess the frequency of variants near RALGAPA1 across different populations, we retrieved SNPs within a $\pm 100-\mathrm{kb}$ region to the gene from 1000G Database. As a result, the MAF of SNPs near the RALGAPA1 locus was higher in a Taiwanese population than that in Caucasians (SNP MAF: $\sim 0.07 v$ s. $\sim 0.001$; S10 Table). Taken together, we suspected that RALGAPA1 is a specific adiposity-susceptibility gene to the Taiwanese population.

\section{Cell types associated with adiposity traits in a Taiwanese population}

To identify critical cell types that are genetically associated with the four adiposity traits in a Taiwanese population, we conducted cell type enrichment tests on Taiwanese GWAS results using active enhancer profiles of 10 cell type categories from the Roadmap project. To avoid confounding due to the variant LD patterns and allelic frequency differences, we first extracted a subset of variants that reached to the suggested significance by LD clumping. Then, we performed a permutation by controlling the MAF of background variants. For BF\%, significant enrichment of variants in active enhancers was observed in the connective bone category $(\mathrm{FDR}=0.05$; Fig 3$)$. Further investigation revealed nominally significant enrichment of active enhancers of all three cell types from the connective bone category (including connective cells, fibroblasts, and osteoblasts; all $p<0.05$; S8 Fig). For the WHR, we detected enrichment in six (of 10) categories (including adipose, cardiovascular, connective and bone, gastrointestinal, liver, and skeletal muscle; with FDRs of $<0.05$ ). In addition, no enrichment of BMI- or WCrelated variants of active enhancers was detected in any of the 10 cell categories.

A previous Japanese study identified the enrichment of the BMI in B cells' active enhancers (a subgroup of the "immune-related" category) [23], however, we are not able to replicate that observation (all nominal $p>0.05$; S9 Fig). Here, we detected nominal significance enrichment 


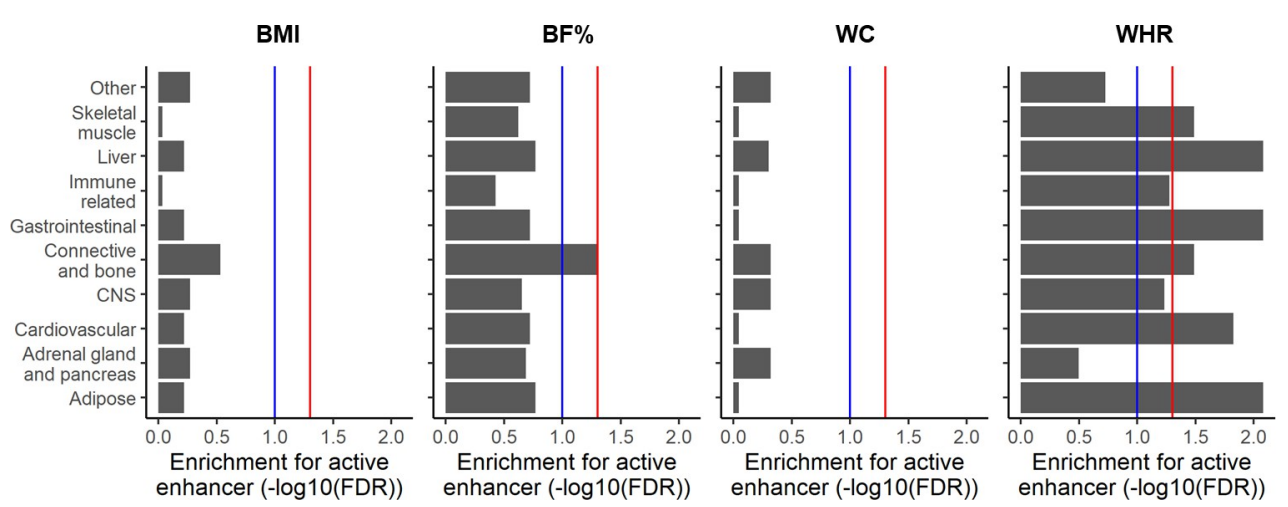

Fig 3. Active enhancer enrichment of obesity-related variants in 10 cell type categories. $p$ values were obtained from 2000 permutations. The false detection rate (FDR) was obtained using the Benjamin-Hochberg method. The red line denotes an FDR of $<0.05$, and blue line denotes an FDR of $<0.1$.

https://doi.org/10.1371/journal.pgen.1009952.g003

of WHR-related variants of active enhancers of the spleen and thymus. Moreover, studies suggested a role of the CNS in the BMI $[29,30]$. However, we found no evidence regarding neuronal implications in the BMI in our Taiwanese population (Fig 3). Subgroup active enhancer enrichment analyses were further conducted by categorizing the CNS category into seven subgroups. As a result, we found significant enrichment (with an FDR of $<0.05$ ) in active enhancers of the anterior caudate, cingulate gyrus, and inferior temporal lobe for BF\% (S10 Fig); and suggested significant (with an FDR of $<0.1$ ) of the middle hippocampus (for BMI and BF\%), anterior caudate (for BMI), anterior caudate (for BMI), cingulate gyrus (for BMI), inferior temporal lobe (for BMI), and angular gyrus (for BF\%). In contrast, enrichment was not detected of any of the seven neuronal subgroups for WC or the WHR.

\section{Variance explained for four adiposity traits by GWAS variants in a Taiwanese population}

The polygenic effects of the four phenotypes were estimated by all GWAS variants in our Taiwanese population-based cohort. Autosomal variants accounted for a proportion of $23.94 \%$ $\pm 1.6 \%$ (standard error of the mean (SEM)) of the phenotypic variance of BMI. This proportion was slightly lower than those of Europeans $(27.4 \% \pm 2.5 \%)$ [31] and Japanese $(29.8 \% \pm 3.4 \%)$ [23]. However, the proportion of variance explained by the BMI was comparable to the UKBB GWAS (24.6\%). For BF\%, WC, and the WHR, estimated phenotypic variances explained by autosomal variants were $18.35 \% \pm 1.7 \%$ ( $v$ s. the UKBB GWAS of $22.0 \%$ ), $18.47 \% \pm 1.6 \%$ ( $v$ s. the UKBB GWAS of $20.4 \%$ ), and $13.41 \% \pm 1.6 \%$ ( $v$ s. Scotland of $24.0 \% \pm 1.0 \%$ [32]), respectively. Overall, we observed a slight decrease in the proportions of variance explained by adiposity traits in a Taiwanese population.

\section{Pathway analysis for the loci associated with adiposity traits}

Using lead variants for each phenotype from Taiwanese subjects, we conducted pathway analyses to identify canonical pathways (CPs) or gene ontology (GO) biological pathways (BPs) for adiposity traits. Using an FDR threshold of 0.05 , we found that variants associated with the BMI were all implicated in neuron-related pathways (Table 4). These results were similar to the previous findings in Europeans [16], i.e., the BMI contains a neuronal component. Surprisingly, we detected neuronal pathway associations for variants associated with $\mathrm{BF} \%$, WC, and the WHR, suggesting neuronal implications in adiposity in the Taiwanese population. For the 
Table 4. Significant pathways (with a false discovery rate $($ FDR) of $<0.05)$ that were associated with adiposity traits.

\begin{tabular}{|c|c|c|}
\hline Trait & Canonical pathways & Gene ontology biological processes \\
\hline \multirow[t]{5}{*}{ BMI } & N.s. & 1. Nervous system development \\
\hline & & 2. Neuron differentiation \\
\hline & & 3. Generation of neurons \\
\hline & & 4. Central nervous system development \\
\hline & & 5. Neurogenesis \\
\hline \multirow[t]{5}{*}{$\mathrm{BF} \%$} & N.s. & 1. Generation of neurons \\
\hline & & 2. Neurogenesis \\
\hline & & 3. Nervous system development \\
\hline & & 4. Central nervous system development \\
\hline & & 5. Neuron differentiation \\
\hline \multirow[t]{8}{*}{ WC } & [Kegg] Axon guidance & 1. Generation of neurons \\
\hline & & 2. Neurogenesis \\
\hline & & 3. Neuron differentiation \\
\hline & & 4. Nervous system development \\
\hline & & 5. Neuron development \\
\hline & & 6. Axonogenesis \\
\hline & & 7. Neurite development \\
\hline & & 8. Cellular morphogenesis during differentiation \\
\hline \multirow[t]{8}{*}{ WHR } & 1. [Reactome] Neuronal system & 1. Anatomical structure development \\
\hline & 2. [Reactome] Axon guidance & \\
\hline & 3. [Reactome] Developmental biology & \\
\hline & 4. [PID] ER nongenomic pathway & \\
\hline & 5. [Reactome] transmission across chemical synapses & \\
\hline & 6. [Reactome] potassium channels & \\
\hline & 7. [Reactome] platelet homeostasis & \\
\hline & 8. [Kegg] glycosaminoglycan degradation & \\
\hline
\end{tabular}

N.s., no significance; [Kegg]: Kyoto Encyclopedia of Genes and Genomes; [Reactome]: Reactome Pathway Database; [PID]: The Pathway Interaction Database. The pathways were ordered by significance (from the most significant to the less significant pathways).

https://doi.org/10.1371/journal.pgen.1009952.t004

WHR, additional pathways were identified, which included an estrogen receptor (non-genomic) pathway, potassium channels, platelet homeostasis, glycoaminoglycan degradation, and a developmental pathway, which implied more-complicated biological regulation of the WHR compared to the $\mathrm{BMI}, \mathrm{BF} \%$, and WC.

\section{Genetic correlation analysis among adiposity traits}

Due to similar results obtained for adiposity phenotypes, we further identified genetic correlations among the BMI, BF\%, WC, and WHR. Our results showed that the BMI exhibited high genetic correlations with $\mathrm{BF} \%$ and $\mathrm{WC}$, indicating that the pleiotropic effects of genes control three phenotypes. For example, WHR showed a modest correlation $\left(\gamma_{\mathrm{g}}=0.5 \sim 0.7\right)$ with BMI, $\mathrm{BF} \%$ and WC, meaning that genes affecting central obesity are not as the same as those affecting general obesity (S11 Fig). Our findings were consistent with previous studies in Asian population (genetic correlation of 0.53 between BMI and WHR) [33] and the National Heart, Lung, and Blood Institute Twin Study (genetic correlation of 0.52 between BMI and WHR) [34]. 


\section{Discussion}

Here, we revealed 13 independent adiposity phenotypes-related trait-loci pairs (seven for BMI, three for $\mathrm{BF} \%$, two for WC, and one for WHR) by integrating medical data and genome-wide SNP profiles from TWB. These associations were successfully replicated in a meta-analysis of Japanese and European populations. Based on our association analysis and LD-based clumping results, FTO loci strongly associated with BMI, BF\% and WC. FTO is widely known to be associated with obesity from previous reports on different ethnic groups [17], and it has been strongly associated with several adiposity phenotypes, such as extreme obesity [35], early-onset obesity [35], the BMI [36], BF\% [24], WC [25], and the WHR [25]. In this study, rs1421085 (located in the FTO locus) was associated with the BMI, BF\% and WC (Tables 2 and 3). Actually, this particular SNP was reported to increase expression of IRX3 and IRX5 through 3D chromatin effects during preadipocyte differentiation, thereby enhancing the formation of energy-storing white adipocytes, lipid storage, and obesity development [37]. Interestingly, when we compared the MAF of rs1421085 in our cohort with those from the UKBB and BBJ cohorts, we found a lower MAF of rs1421085 (of 0.126) in the Taiwanese population than that in European (MAF of 0.402) and Japanese (MAF of 0.201) populations. Notably, we found no significant associations between the FTO gene and the WHR trait in our study population. Since the top BMI variant (exemplified by rs11642015) in the FTO gene showed a $p$-value of $1.842 \times 10^{-5}$ in WHR (TWB data), we denoted that this finding may be due to lack of statistical power. Other loci that were significantly associated with the BMI in this study including rs13130484 on 4p12 [23,38], rs141473007 on 6p12.3 [17], and rs72982988 on 18q21.32 [17,23], which were also significantly associated with obesity in European and East Asian populations. By comparing the SNPs identified here with cataloged GWAS reports, we evaluate the pleiotropic effects of genes on adiposity phenotypes. Four loci were associated with multiple adiposity phenotypes, such as BMI, early-onset extreme obesity, fat body mass, and WC, among others (S3 Table). These results suggested that pleiotropic effects of some genes lead to several adiposity phenotypes. For example, rs 109383397 may simultaneously impact BMI and BF\%. Moreover, loci in FTO (e.g., rs11642015 and rs1558902) exhibited pleiotropic effects on T2DM and metabolic syndrome, indicating that these loci may have effects on obesity-related comorbidities. In line with these findings, a genetic correlation analysis revealed strong similarities among $\mathrm{BMI}, \mathrm{BF} \%$ and WC phenotypes, suggesting that the genes-mediated phenotypes are highly correlated. Unexpectedly, WC, an indicator of abdominal obesity, showed higher genetic correlations with $\mathrm{BMI}\left(\gamma_{\mathrm{g}}=0.88\right)$ and $\mathrm{BF} \%\left(\gamma_{\mathrm{g}}=0.9\right)$; meanwhile, another indicator of abdominal obesity, WHR, showed a moderate genetic correlation with BMI $\left(\gamma_{\mathrm{g}}=0.52\right)$ but a high genetic correlation with WC $\left(\gamma_{\mathrm{g}}=0.7\right)$, consistent with the findings of a previous study [39]. Notably, we observed higher correlations among $\mathrm{BMI}, \mathrm{BF} \%$ and $\mathrm{WC}$, and relatively lower genetic correlations with WHR. This observation was further supported by the pathway analysis, which revealed that $\mathrm{BMI}, \mathrm{BF} \%$ and $\mathrm{WC}$ were only associated with neural processes; however, in WHR was associated with pathways including estrogen receptor (non-genomic) signaling, potassium channels, platelet homeostasis, glycoaminoglycan degradation, and a developmental pathway.

In this study, we found that the RALGAPA1 locus are specifically associated with BMI in the Taiwanese cohort. SNPs in RALGAPA1 were successfully replicated in a meta-analysis on East Asian (BBJ cohort) and multi-ethnic populations (GERA cohort); 92 SNPs were with the suggested significance level in the \pm 100 -kb region around RALGAPA1. We also examined the cis-expression quantitative trait locus (cis-eQTL) data of the most significant SNP, rs8004796, and found the $c i s-\mathrm{eQTL}$ in adipose-visceral tissues $(p=0.0032$; FDR $=0.000053)$. Aside from the cis-eQTL evidence, we also found $\mathrm{H} 3 \mathrm{~K} 4 \mathrm{me} 3$ histone modifications and a transcription factor-binding site (RBM22) at rs8004796 by using UCSC genome browser. However, there was 
no evidence of transcriptional activities in a cell line (S12 Fig). We further examined another SNP, rs76115093, which exhibited high LD with rs8004796 $\left(r^{2}=1\right)$. Indeed, several transcription factor-binding sites were predicted around rs76115093. The functional experiments showed the activity of a CCCTC-binding factor (CTCF), therefore, we considered rs76115093 to be a potentially functional variant that might alter RALGAPA1 expression via a $3 \mathrm{D}$ interaction with the promoter region (S13 Fig) [40]. These results imply that fluctuations in gene expression levels may be linked to the rs 8004796 polymorphism. Furthermore, MAFs of SNPs in RALGAPA1 were low (S7 Table). We considered the association between this gene and $B M I$ to be specific to the Taiwanese population. Previous studies reported that overexpression of the RALGAPA1 mutant protein (Thr735Ala) decreases RalA protein activation, and it attenuates RalA-dependent glucose transporter 4 (GLUT4) and cluster of differentiation 36 (CD36) translocation in response to insulin stimulation in skeletal muscle cells [41]. Thus, further testing will be needed to determine whether the RALGAPA1 is associated with obesity in an independent Taiwanese cohort.

A heritability analysis revealed a slight decrease in the proportions of variance explained by all four adiposity traits in the Taiwanese population compared to that in Europeans and Japanese populations. These results confirmed the existence of genetic predispositions to adiposity traits and highlighted the importance of environmental determinants. Based on these findings, it is most reasonable to consider environmental interventions (healthy lifestyle promotions, weight-loss plans, promotion of regular exercise, etc.) for managing or controlling adiposity in different populations. In accordance with this point of view, Lin et al., reported that regular exercise (especially regular jogging) can diminish BMI, BF\%, WC and HC [42]. Importantly, promotion of regular physical exercise is most salient to Taiwanese subjects with a greater genetic predisposition to adiposity.

A previous study on BMI-associated genes in a European population nicely showed enrichments in central nervous system-related processes, including the involvement of neurotrophin signaling pathway, synaptic function, and glutamate signaling [16]. On the other hand, ethanol oxidation and glycolysis gluconeogenesis pathways were identified in an East Asian population [23]. For WC and WHR phenotypes (both BMI-adjusted), genes identified in Europeans were found to be significantly enriched in adipose-related tissues, adiponectin signaling and insulin sensitivity [25], whereas the genes in East Asians were enriched in corticotropin-releasing hormone signaling and gonadotropin-releasing hormone $(\mathrm{GnRH})$ signaling pathways [18]. These results suggest a need to assess multiple populations in order to better understand the pathways underlying adiposity. In the current study, we found that all loci associated with the four adiposity traits were significantly enriched in neuron-related pathways (Table 4), however, these pathways were not reported to be associated with $\mathrm{BF} \%$, WC or WHR in previous studies. For $\mathrm{BF} \%$ in particular, this finding was further supported by results of an active enhancer enrichment analysis (S10 Fig), which showed significant enrichment in active enhancers of BF $\%$-associated loci for the anterior caudate, cingulate gyrus, and inferior temporal lobe. We also found enrichment of WHR-associated genes in a developmental pathway, which is consistent with previous findings [25]. Thus, our results revealed adiposity-associated pathways that have particularly pronounced effects on adiposity in a Taiwanese population.

There are some limitations in this study. First, we did not observe a GWAS signal for WHR in our cohort (before the meta-analysis), probably because the sample size used for the analysis was not enough to obtain sufficient power. Second, we accounted for age, age ${ }^{2}$, sex, and the top 10 PCs of ancestry as covariates, whereas many other environmental factors may be associated with adiposity phenotypes, such as current medications, physical activity levels, smoking behavior, and alcohol usage. Gene-environment effects should be considered and estimated in future studies. 
In this study, we performed a GWAS in a Taiwanese population and identified 13 significant adiposity-associated trait-locus pairs. We revealed two BF\% loci (GNPDA2-GABRG1 [4p12] and RNU6-2-PIAS1 [15q23]) that were pleiotropic across adiposity-related traits and uncovered a BMI-associated locus in RALGAPA1, which is specific to the Taiwanese population. A heritability analysis revealed a slightly lower than expected variance of adiposity traits that could be explained by genetic predispositions, suggesting the importance of behavioral interventions to improve adiposity. Finally, our results revealed the critical role of neural effects on BF\%, WC and WHR.

\section{Materials and methods}

\section{Ethics statement}

Ethical approval of the study was granted by the Institutional Review Board (IRB no. N201802059) of Taipei Medical University and TWB, Academia Sinica (TWBR10505-05, TWBR10602-02, TWBR10703-02, and AS-IRB01-16018) before conducting the study.

\section{Study population}

This study included 23,996 subjects with complete information of age, sex, BMI, and whole genotype data from the Taiwan Biobank (TWB). To date, 114,533 participants have been recruited from the community, containing a total of 27,700 genome-wide array genotyping data among them. The main aim of the TWB is to provide researchers with health information of Taiwanese people so that they can improve health-related problems concerning common chronic diseases. Previously, Chen et al. [43] and Lo et al. [44] demonstrated that $~ 80 \%$ of Han Taiwanese are genetically closer to the Southern Han ancestry in China. However, since the study subjects were all collected from the Taiwanese cohort, we use the term "Taiwanese" for precision sake.

Local community participants that are included were aged 30 70 years, were physically active, and were self-reported to be of Taiwanese Han-Chinese ancestry; specimens, blood, urine, detailed questionnaires, and data of a physical examination were collected. Written informed consent was obtained from all the individuals while participating in the TWB project. Participants were all genotyped by a custom Axiom Genome-Wide Array Plate, that contained 646,973 single-nucleotide polymorphism (SNP) sites, called the TWB chip 1, based on technology developed by Affymetrix. Individuals with cancer or a cancer history, who were non-residents of Taiwan, or who withdrew were excluded. In total, 21,978 subjects were included for a subsequent analysis after quality control.

\section{Data quality control steps and imputation}

For sample quality control of the GWAS, samples with ambiguous sex data (by sex check) were excluded; individuals with a call rate of $<0.95$, related individuals (PIHAT $>0.1875$ ), and those with a heterozygosity rate of greater than the mean \pm 5 standard deviations (SDs); calculated by PLINK v1.9 [45]) were further excluded. Moreover, all samples passed the ancestry check. For genotypic quality control, SNPs with a call rate of $>0.05$, Hardy-Weinberg equilibrium (HWE) $p$-value of $<1 \times 10^{-5}$ (calculated using only control subset), and a minor allele frequency $(\mathrm{MAF})<0.05$ were further discarded.

Genotype imputation was performed using SHAPEIT (v2.r790) [46] and IMPUTE2 (v2.3.1) [47], with a reference panel compiled by a Taiwanese population (TWB) and 1000G East Asian (EAS) population whole-genome sequencing (WGS) data. After imputation, multiallelic sites and SNPs with a post-imputation call rate of $>0.05$, a MAF of $<0.01$, or $R^{2}<0.3$ 
were excluded. The final set of 6,546,460 variants were prepared for the following association analysis.

To assess the population stratification of our Taiwanese population, we merged the data using $~ 60,000$ SNPs (with low linkage disequilibrium (LD) $\left(r^{2}<0.2\right)$ and MAFs of $<0.05$ ) to 1 KGP data with 26 populations. Principal component analysis (PCA) was then conducted using GCTA [48]. As no individuals were outside of the East Asian cluster, we included all individuals in subsequent analyses.

\section{Phenotype preparation}

We collected data (height, weight, WC, hip circumference (HC), and BF\%) from physical examination data in the TWB. The BMI was calculated as the weight $(\mathrm{kg})$ divided by the height squared $\left(\mathrm{m}^{2}\right) ; \mathrm{BF} \%$ was estimated by a bioelectrical impedance analysis (BIA); the WHR was a comparison of WC $(\mathrm{cm})$ and $\mathrm{HC}(\mathrm{cm})$. Outliers were defined as a phenotypic value beyond the mean \pm 4 SDs. There were 21,930 individuals with BMI data; 21,304 individuals with BF\% data; 21,949 individuals with WC data; and 21,972 individuals with WHR data. All phenotypes (BMI, BF\%, WC, and WHR) were further standardized using a rank-based inverse transformation method because of right-skewed distributions. To do this, we first obtained residuals for log-transformed phenotypes using a linear regression analysis which was adjusted for age, age $^{2}$, sex, and top 10 PCs of ancestry. Then, we transformed the residuals via a rank-based inverse transformation.

\section{Association analysis}

An SNP-based association analysis was performed using PLINK (v1.9) [45] based on linear regression. Owing to the previous adjustment of age, age ${ }^{2}$, sex, and top 10 PCs of ancestry during phenotype preparation, we did not perform any further adjustment in the association analysis. Manhattan plots and quantile-quantile $(\mathrm{Q}-\mathrm{Q})$ plots were generated with the qqman package.

\section{LD score regression analysis}

The LD score intercepts of the four adiposity traits in Taiwanese population were calculated using the LDSC package [49].

\section{Conditional analysis and meta-analysis}

A conditional analysis was conducted using GCTA [50] to determine independent signals near the most significantly associated loci ( $\pm 1-\mathrm{Mb}$ region). LD information was calculated using imputed data of all individuals, and SNPs with collinearity of $>0.9$ were excluded. We applied LocusZoom to generate regional association plots [51]. A meta-analysis was performed using the inverse-variance fixed-effect method implemented in METAL software [52] with no further genomic control (GC) correction. Heterogeneity between studies was calculated with Cochran's $Q$ test. Successful replication $p$ was designated at $5 \times 10^{-8}$, with directions consistent between the discovery and replication datasets.

Summary statistics were downloaded from the GWAS catalog [53], GIANT consortium, and UK Biobank (UKBB) as follows: (i) The GIANT consortium. For WC and WHR, we retrieved the data from Shungin, D. et al. [25] that contained 224,459 individuals. In total, 1109 and 544 signals for WC (top variant: rs1558902; FTO gene) and WHR (top variant: rs1121980; FTO gene) were identified in the study, respectively. (ii) GWAS catalog. Rankbased inverse transformed BMI data from a Japanese cohort conducted by Akiyama et al. [23] 
were used in our study. This cohort contained 173,140 Japanese individuals (Biobank Japan; BBJ) and 5,961,601 variants. In total, 5903 variants reached the genome-wide significance threshold, with 112 novel loci against the previous studies being identified. The strongest signal was rs11642015 on the FTO gene. A multi-ethnic BMI (rank-based inverse transformed) cohort [17] contained 334,487 individuals (non-Hispanic whites, Latinos, East Asians, African Americans and South Asians) was also included in this study. With 10,694 genome-wide significant signals identified, 30 novel loci were further identified. The most significant locus was rs1421085 on the FTO gene. Additionally, BF\% data was obtained from a study conducted by Hübel C et al. [24], which contains 155,961 Europeans. In this study, 5,056 variants reached the genome-wide significant threshold, with the strongest signal be rs1421085 on the FTO gene. (iii) The UK Biobank. Summary statistics of four adiposity-related traits (rank-based inverse transformed; 361,194 samples for each phenotype) were acquired from Neale's lab website (http://www.nealelab.is/uk-biobank). The number of significant loci in BMI, BF\%, WC and WHR were $39,879,39,879,31,847$ and 35,029, respectively. For BMI, the most significant signal was rs62048402 (FTO gene). For BF\% and WC, the top signal was rs11642015 (FTO gene). For WHR, the top signal was rs577721086 (FTO gene). Due to the incompleteness of genotype information in WHR, we thus directly compared the regression $\beta$ values between WHR summary statistics and our suggestive significant loci using Spearman's correlation test.

\section{Functional annotations for variants}

Independent significant SNPs with each other at $r^{2}<0.1$ and SNPs with high LD of these SNPs $\left(r^{2} \geq 0.6\right)$ were mapped to genes in FUMA v1.3.4 [54] using the following strategies. (1) Positional mapping. FUMA performed positional mapping based on SNP annotation from ANNOVAR [55] using a default physical distance (of $\pm 10 \mathrm{~kb}$ ). Then the lists of SNPs were further mapped with the Combined Annotation-Dependent Depletion (CADD) score. (2) Chromatin state mapping. To investigate the potential regulatory functions of our loci, we applied chromatin state mapping based on the 15-core ChromHMM model. The chromatin state with 15 categorical states represented the genome accessibility predicted by ChromHMM based on epigenomic data of five chromatin marks (H3K4me3, H3K4me1, H3K36me3, H3K27me3, and $\mathrm{H} 3 \mathrm{~K} 9 \mathrm{me} 3$ ) for 127 cell types. (3) 3D chromatin interaction mapping. FUMA maps SNPs to genes based on Hi-C data (GSE87112) of 21 tissues/cell types [56]. The default threshold of the false discovery rate (FDR; $p<10^{-6}$ ) was set to define significant interactions.

\section{Cell type specificity analysis}

Active enhancer profiles of different cell types in the ChromHMM 18-state model (state 9) were acquired from the Roadmap project. We first grouped cell types into 10 cell type categories for analysis. Clumped SNP lists of the four adiposity traits were subjected to active enhancer enrichment tests. The test was conducted by comparing the number of overlapping clumped variants in active enhancers to the number of overlapping randomly selected variants in active enhancers. To avoid bias from LD, the background SNP list was pruned based on LD information of Taiwanese population genomic profiles. To prevent confounding from allele frequency differences, a background gene list was sampled based on allele frequency categories. The allele frequency categories were as follows: (1) category $1: \mathrm{MAF} \leq 5 \%$; (2) category 2 : $5 \%<\mathrm{MAF} \leq 15 \%$; (3) category $3: 15 \%<\mathrm{MAF} \leq 30 \%$; and (4) category 4 : MAF $>30 \%$. For each test, we repeated the sampling procedure 2000 times (permutations) to calculate $p$ values. The FDR was finally estimated using the Benjamini-Hochberg (B-H) method. 


\section{Heritability analysis}

To determine the proportion of variance explained (heritability) with autosomal variants, we first generated a genetic relationship matrix (GRM) using genome-wide complex trait analysis (GCTA)-genomic restricted maximum likelihood (GREML) method. The log-transformed phenotype value was transformed to a $Z$ score and adjusted for age, age ${ }^{2}$, and sex. An estimate of the variance of adiposity phenotypes was then calculated using the "reml" function implemented in GCTA with parameter "-grm-cutoff 0.05".

\section{Pathway analysis}

To determine which mechanisms are involved in regulating adiposity on our gene lists, we conducted a pathway analysis using the snpGeneSets package. The annotation file (Homo_sapiens.GRCh37.87.gtf) for SNP-to-gene mapping was acquired from the website $\mathrm{ftp}: / / \mathrm{ftp}$. ensembl.org/pub/grch37/current/gtf/homo_sapiens/. In this study, the SNP-to-gene mapping step was conducted by setting the flanking region to $2000 \mathrm{bp}$.

\section{Genetic correlations and pleiotropic analysis}

To estimate genetic correlations $\left(\gamma_{\mathrm{g}}\right)$ between adiposity phenotypes, a bivariate LD score regression [57] was conducted on the current GWAS. All of our summary statistics were transformed to a standard format, and LD information was calculated from the $1000 \mathrm{G}$ phase 3 East Asian panel (v2.2). The $p$ value of each pair of genetic correlations ( $p$ value for $\gamma_{\mathrm{g}}$ ) was then adjusted for the false discovery rate (FDR) for multiple corrections. We further compared our suggested SNP lists with the GWAS catalog to examine pleiotropic effects.

\section{Supporting information}

S1 Fig. Schematic showing the flowchart quality control, association analysis and postGWAS steps in this study. (PDF)

S2 Fig. Principle component analysis plots examining population stratification. Substructure for (a) our Taiwanese cohort (TWN) and 1000 Genome and (b) our Taiwanese cohort (TWN) and East Asians. Graphs represent the first two principal components. Different colors illustrate each sub-population. ACB, African Caribbean in Barbados; ASW, African ancestry in Southwest USA; BEB, Bengali in Bangladesh; CDX, Chinese Dai in Xishuangbanna, China; CEU, Utah residents with Northern and Western European ancestry from the CEPH collection; CHB, Han Chinese in Beijing; CHD, Chinese in Metropolitan Denver; CHS, Han Chinese South; CLM, Colombian in Medellin, Colombia; ESN, Esan in Nigeria; FIN, Finnish in Finland; GBR, British in England and Scotland; GIH, Gujarati Indians in Houston; GWD, Gambian in Western Division, The Gambia-Mandinka; IBS, Iberian populations in Spain; ITU, Indian Telugu in the UK; JPT, Japanese in Tokyo; KHV, Kinh in Ho Chi Minh City, Vietnam; LWK, Luhya in Webuye, Kenya; MXL, Mexican Ancestry in Los Angeles, California; MSL, Mende in Sierra Leone; PEL, Peruvian in Lima, Peru; PJL, Punjabi in Lahore, Pakistan; PUR, Puerto Rican in Puerto Rico; STU, Sri Lankan Tamil in the UK; TSI, Toscani in Italia; TWN, Taiwanese; YRI, Yoruba in Ibadan.

(PDF)

S3 Fig. Quantile-quantile plots of adiposity phenotypes. BMI, body-mass index; BF\% body fat percentage; WC, waist circumference; WHR, waist-hip ratio.

(PDF) 
S4 Fig. Significant loci of adiposity phenotypes identified in a Taiwanese population. Regional plots before (left) and after (right) a conditional analysis. hg19 of 1000 Genome East Asian version (Nov. 2014) was used as the reference panel. BMI, body-mass index; BF\%, body fat percentage; WC, waist circumference. (PDF)

S5 Fig. Functional annotation of extended single-nucleotide polymorphism (SNP) lists of four phenotypes. Functional annotation results from positional mapping by ANNOVAR. The number of extended SNPs of each phenotype is listed in the figure legend. BMI, body-mass index; $\mathrm{BF} \%$, body fat percentage; WC, waist circumference; WHR, waist-hip ratio. (PDF)

S6 Fig. Proportions of the open state across different tissue types in the 15-core ChromHMM state model. The figure shows that the proportions of open state (chromHMM state $<7)$ are similar across all tissues. The 127 epigenomes from the 15 -core chromatin state model were sorted into 29 tissue categories.

(PDF)

S7 Fig. Functional annotations of RALGAPA1 loci for the four adiposity traits. (PDF)

S8 Fig. Subgroup active enhancer enrichment of adiposity variants in (left) connective cells; (middle) fibroblasts; and (right) osteoblasts ("connective and bone" category). The red line denotes $p<0.05$, and the blue line denotes $p<0.1$.

S9 Fig. Subgroup active enhancer enrichment of adiposity variants in B cells, T cells, the spleen, and thymus ("immune related" category). The red line denotes $p<0.05$, and the blue line denotes $p<0.1$.

(PDF)

S10 Fig. Active enhancer enrichment of neuronal subgroups for the four adiposity traits. (PDF)

S11 Fig. Pairwise genetic correlations of four adiposity-related traits. (PDF)

S12 Fig. Epigenetic profile of rs8004796 on the UCSC genome browser. A transcriptional factor-binding site, RBM22, was predicted to bind to rs8004796. However, there were no transcriptional activities in the cell lines.

(PDF)

S13 Fig. Epigenetic profile of rs76115093 on the UCSC genome browser. rs76115093 showed evidence of histone modification and transcriptional factor binding, and additionally, CTCF modulation was shown in different cell lines. rs76115093 may be a functional variant. (PDF)

S1 Table. Summary statistics of body mass index (BMI) in Taiwanese cohort.

S2 Table. Summary statistics of body fat percentage (BF\%) in Taiwanese cohort.

S3 Table. Summary statistics of waist circumference (WC) in Taiwanese cohort. (LRZ) 
S4 Table. Summary statistics of waist-hip ratio (WHR) in Taiwanese cohort. (LRZ)

S5 Table. Summary of the results of a trans-ethnic meta-analysis. (PDF)

S6 Table. Pleiotropy of the 13 adiposity trait-associated loci in a Taiwanese population. (PDF)

S7 Table. Frequencies of novel body fat percentage (BF\%)-associated single-nucleotide polymorphisms (SNPs) in different populations.

S8 Table. Single-nucleotide polymorphisms (SNPs) in high linkage disequilibrium (LD) $\left(r^{2}>0.8\right)$ to novel body fat percentage (BF\%)-associated variants. (PDF)

S9 Table. Functional annotations of single-nucleotide polymorphisms (SNPs) using the HaploReg and RegulomeDB databases.

S10 Table. Comparison of the minimum allele frequency (MAF) of single-nucleotide polymorphisms (SNPs) on RALGAPA1 ( $\pm 100-\mathrm{kb}$ region) in $1000 \mathrm{G}$ populations.

(PDF)

\section{Author Contributions}

Conceptualization: Henry Sung-Ching Wong, Wei-Chiao Chang.

Data curation: Henry Sung-Ching Wong, Szu-Yi Tsai, Hou-Wei Chu.

Formal analysis: Henry Sung-Ching Wong, Szu-Yi Tsai, Min-Rou Lin, Gan-Hong Lin, YuTing Tai.

Funding acquisition: Wei-Chiao Chang.

Investigation: Henry Sung-Ching Wong, Hou-Wei Chu, Chen-Yang Shen, Wei-Chiao Chang.

Methodology: Henry Sung-Ching Wong, Szu-Yi Tsai, Hou-Wei Chu.

Project administration: Wei-Chiao Chang.

Resources: Chen-Yang Shen, Wei-Chiao Chang.

Supervision: Chen-Yang Shen, Wei-Chiao Chang.

Validation: Henry Sung-Ching Wong, Szu-Yi Tsai, Chen-Yang Shen.

Visualization: Henry Sung-Ching Wong, Szu-Yi Tsai.

Writing - original draft: Henry Sung-Ching Wong, Szu-Yi Tsai, Wei-Chiao Chang.

Writing - review \& editing: Henry Sung-Ching Wong, Wei-Chiao Chang.

\section{References}

1. Mi Y-J, Zhang B, Wang H-J, Yan J, Han W, Zhao J, et al. Prevalence and Secular Trends in Obesity Among Chinese Adults, 1991-2011. American Journal of Preventive Medicine. 2015; 49(5):661-9. https://doi.org/10.1016/j.amepre.2015.05.005 PMID: 26275960 
2. Abarca-Gómez L, Abdeen ZA, Hamid ZA, Abu-Rmeileh NM, Acosta-Cazares B, Acuin C, et al. Worldwide trends in body-mass index, underweight, overweight, and obesity from 1975 to 2016: a pooled analysis of 2416 population-based measurement studies in 128.9 million children, adolescents, and adults. The Lancet. 2017; 390(10113):2627-42. https://doi.org/10.1016/s0140-6736(17)32129-3 PMID: 29029897

3. Trends in adult body-mass index in 200 countries from 1975 to 2014 : a pooled analysis of 1698 population-based measurement studies with 19.2 million participants. The Lancet. 2016; 387(10026):137796. https://doi.org/10.1016/S0140-6736(16)30054-X PMID: 27115820

4. Chang HC, Yang HC, Chang HY, Yeh CJ, Chen HH, Huang KC, et al. Morbid obesity in Taiwan: Prevalence, trends, associated social demographics, and lifestyle factors. PLoS One. 2017; 12(2):e0169577. Epub 2017/02/06. https://doi.org/10.1371/journal.pone.0169577 PMID: 28152059; PubMed Central PMCID: PMC5289420.

5. Czernichow S, Kengne AP, Stamatakis E, Hamer M, Batty GD. Body mass index, waist circumference and waist-hip ratio: which is the better discriminator of cardiovascular disease mortality risk?: evidence from an individual-participant meta-analysis of 82864 participants from nine cohort studies. Obes Rev. 2011; 12(9):680-7. Epub 2011/04/28. https://doi.org/10.1111/j.1467-789X.2011.00879.x PMID: 21521449; PubMed Central PMCID: PMC4170776.

6. Roka R, Michimi A, Macy G. Associations Between Hypertension and Body Mass Index and Waist Circumference in U.S. Adults: A Comparative Analysis by Gender. High Blood Press Cardiovasc Prev. 2015; 22(3):265-73. https://doi.org/10.1007/s40292-015-0106-3 PMID: 26014837.

7. Guh DP, Zhang W, Bansback N, Amarsi Z, Birmingham CL, Anis AH. The incidence of co-morbidities related to obesity and overweight: a systematic review and meta-analysis. BMC Public Health. 2009; 9:88. Epub 2009/03/27. https://doi.org/10.1186/1471-2458-9-88 PMID: 19320986; PubMed Central PMCID: PMC2667420.

8. Qiao $Q$, Nyamdorj $R$. Is the association of type II diabetes with waist circumference or waist-to-hip ratio stronger than that with body mass index? European Journal Of Clinical Nutrition. 2009; 64:30. https:// doi.org/10.1038/ejcn.2009.93 https://www.nature.com/articles/ejcn200993\#supplementaryinformation. PMID: 19724291

9. Lauby-Secretan B, Scoccianti C, Loomis D, Grosse Y, Bianchini F, Straif K. Body Fatness and Cancer —Viewpoint of the IARC Working Group. New England Journal of Medicine. 2016; 375(8):794-8. https://doi.org/10.1056/NEJMsr1606602 PMID: 27557308.

10. Tang B, Han C-T, Zhang G-M, Zhang C-Z, Yang W-Y, Shen Y, et al. Waist-hip Ratio (WHR), a Better Predictor for Prostate Cancer than Body Mass Index (BMI): Results from a Chinese Hospital-based Biopsy Cohort. Scientific Reports. 2017; 7:43551. https://doi.org/10.1038/srep43551 https://www. nature.com/articles/srep43551\#supplementary-information. PMID: 28272469

11. López-Hernández D. Epidemiological Association Between Body Fat Percentage and Cervical Cancer: A Cross-sectional Population-based Survey from Mexico. Archives of Medical Research. 2013; 44 (6):454-8. https://doi.org/10.1016/j.arcmed.2013.08.007 PMID: 24051040

12. Elks CE, den Hoed M, Zhao JH, Sharp SJ, Wareham NJ, Loos RJ, et al. Variability in the heritability of body mass index: a systematic review and meta-regression. Front Endocrinol (Lausanne). 2012; 3:29. Epub 2012/05/31. https://doi.org/10.3389/fendo.2012.00029 PMID: 22645519; PubMed Central PMCID: PMC3355836.

13. Lee J, Chen L, Snieder H, Chen DF, Lee LM, Liu GF, et al. Heritability of obesity-related phenotypes and association with adiponectin gene polymorphisms in the Chinese national twin registry. Ann Hum Genet. 2010; 74(2):146-54. Epub 2010/03/06. https://doi.org/10.1111/j.1469-1809.2010.00565.x PMID: 20201938.

14. Hsu F-C, Lenchik L, Nicklas BJ, Lohman K, Register TC, Mychaleckyj J, et al. Heritability of Body Composition Measured by DXA in the Diabetes Heart Study. Obesity Research. 2005; 13(2):312-9. https:// doi.org/10.1038/oby.2005.42 PMID: 15800289

15. Tarnoki AD, Tarnoki DL, Medda E, Cotichini R, Stazi MA, Fagnani C, et al. Bioimpedance analysis of body composition in an international twin cohort. Obes Res Clin Pract. 2014; 8(3):e201-98. Epub 2014/ 05/23. https://doi.org/10.1016/j.orcp.2012.09.001 PMID: 24847671.

16. Locke AE, Kahali B, Berndt SI, Justice AE, Pers TH, Day FR, et al. Genetic studies of body mass index yield new insights for obesity biology. Nature. 2015; 518(7538):197-206. Epub 2015/02/13. https://doi. org/10.1038/nature14177 PMID: 25673413; PubMed Central PMCID: PMC4382211.

17. Hoffmann TJ, Choquet H, Yin J, Banda Y, Kvale MN, Glymour M, et al. A Large Multiethnic GenomeWide Association Study of Adult Body Mass Index Identifies Novel Loci. Genetics. 2018; 210(2):499515. Epub 2018/08/16. https://doi.org/10.1534/genetics.118.301479 PMID: 30108127; PubMed Central PMCID: PMC6216593. 
18. Wen W, Kato N, Hwang JY, Guo X, Tabara Y, Li H, et al. Genome-wide association studies in East Asians identify new loci for waist-hip ratio and waist circumference. Sci Rep. 2016; 6:17958. Epub 2016/01/21. https://doi.org/10.1038/srep17958 PMID: 26785701; PubMed Central PMCID: PMC4726286.

19. Sung YJ, Pérusse L, Sarzynski MA, Fornage M, Sidney S, Sternfeld B, et al. Genome-wide association studies suggest sex-specific loci associated with abdominal and visceral fat. International Journal Of Obesity. 2015; 40:662. https://doi.org/10.1038/ijo.2015.217 https://www.nature.com/articles/ ijo2015217\#supplementary-information. PMID: 26480920

20. Chu AY, Deng X, Fisher VA, Drong A, Zhang Y, Feitosa MF, et al. Multiethnic genome-wide meta-analysis of ectopic fat depots identifies loci associated with adipocyte development and differentiation. Nature genetics. 2016; 49:125. https://doi.org/10.1038/ng.3738 https://www.nature.com/articles/ng. 3738\#supplementary-information. PMID: 27918534

21. Salinas YD, Wang L, DeWan AT. Multiethnic genome-wide association study identifies ethnic-specific associations with body mass index in Hispanics and African Americans. BMC Genetics. 2016; 17(1):78. https://doi.org/10.1186/s12863-016-0387-0 PMID: 27296613

22. Justice AE, Winkler TW, Feitosa MF, Graff M, Fisher VA, Young K, et al. Genome-wide meta-analysis of 241,258 adults accounting for smoking behaviour identifies novel loci for obesity traits. Nature communications. 2017; 8:14977. https://doi.org/10.1038/ncomms14977 https://www.nature.com/articles/ ncomms14977\#supplementary-information. PMID: 28443625

23. Akiyama M, Okada $Y$, Kanai M, Takahashi A, Momozawa $Y$, Ikeda $M$, et al. Genome-wide association study identifies 112 new loci for body mass index in the Japanese population. Nature genetics. 2017; 49 (10):1458-67. Epub 2017/09/12. https://doi.org/10.1038/ng.3951 PMID: 28892062.

24. Hübel C, Gaspar HA, Coleman JRI, Finucane H, Purves KL, Hanscombe KB, et al. Genomics of body fat percentage may contribute to sex bias in anorexia nervosa. Am J Med Genet B Neuropsychiatr Genet. 2018. https://doi.org/10.1002/ajmg.b.32709 PMID: 30593698

25. Shungin D, Winkler TW, Croteau-Chonka DC, Ferreira T, Locke AE, Magi R, et al. New genetic loci link adipose and insulin biology to body fat distribution. Nature. 2015; 518(7538):187-96. Epub 2015/02/13. https://doi.org/10.1038/nature14132 PMID: 25673412; PubMed Central PMCID: PMC4338562.

26. Graff M, Justice AE, Young KL, Marouli E, Zhang X, Fine RS, et al. Discovery and fine-mapping of height loci via high-density imputation of GWASs in individuals of African ancestry. Am J Hum Genet. 2021; 108(4):564-82. Epub 2021/03/14. https://doi.org/10.1016/j.ajhg.2021.02.011 PMID: 33713608; PubMed Central PMCID: PMC8059339.

27. Ward LD, Kellis M. HaploReg v4: systematic mining of putative causal variants, cell types, regulators and target genes for human complex traits and disease. Nucleic acids research. 2016; 44(D1):D87781. Epub 2015/12/15. https://doi.org/10.1093/nar/gkv1340 PMID: 26657631; PubMed Central PMCID: PMC4702929.

28. Boyle AP, Hong EL, Hariharan M, Cheng Y, Schaub MA, Kasowski M, et al. Annotation of functional variation in personal genomes using RegulomeDB. Genome research. 2012; 22(9):1790-7. Epub 2012/ 09/08. https://doi.org/10.1101/gr.137323.112 PMID: 22955989; PubMed Central PMCID: PMC3431494.

29. Speliotes EK, Willer CJ, Berndt SI, Monda KL, Thorleifsson G, Jackson AU, et al. Association analyses of 249,796 individuals reveal 18 new loci associated with body mass index. Nature genetics. 2010; 42 (11):937-48. Epub 2010/10/12. https://doi.org/10.1038/ng.686 PMID: 20935630; PubMed Central PMCID: PMC3014648.

30. Willer CJ, Speliotes EK, Loos RJ, Li S, Lindgren CM, Heid IM, et al. Six new loci associated with body mass index highlight a neuronal influence on body weight regulation. Nature genetics. 2009; 41(1):2534. Epub 2008/12/17. https://doi.org/10.1038/ng.287 PMID: 19079261; PubMed Central PMCID: PMC2695662.

31. Yang J, Bakshi A, Zhu Z, Hemani G, Vinkhuyzen AA, Lee SH, et al. Genetic variance estimation with imputed variants finds negligible missing heritability for human height and body mass index. Nature genetics. 2015; 47(10):1114-20. Epub 2015/09/01. https://doi.org/10.1038/ng.3390 PMID: 26323059; PubMed Central PMCID: PMC4589513.

32. Nagy R, Boutin TS, Marten J, Huffman JE, Kerr SM, Campbell A, et al. Exploration of haplotype research consortium imputation for genome-wide association studies in 20,032 Generation Scotland participants. Genome medicine. 2017; 9(1):23. Epub 2017/03/09. https://doi.org/10.1186/s13073-0170414-4 PMID: 28270201; PubMed Central PMCID: PMC5339960.

33. Wu Y, Duan H, Tian X, Xu C, Wang W, Jiang W, et al. Genetics of Obesity Traits: A Bivariate GenomeWide Association Analysis. Front Genet. 2018; 9:179. Epub 2018/06/06. https://doi.org/10.3389/fgene. 2018.00179 PMID: 29868124; PubMed Central PMCID: PMC5964872. 
34. Cardon LR, Carmelli D, Fabsitz RR, Reed T. Genetic and environmental correlations between obesity and body fat distribution in adult male twins. Hum Biol. 1994; 66(3):465-79. Epub 1994/06/01. PMID: 8026816

35. Wheeler E, Huang N, Bochukova EG, Keogh JM, Lindsay S, Garg S, et al. Genome-wide SNP and CNV analysis identifies common and low-frequency variants associated with severe early-onset obesity. Nature genetics. 2013; 45:513. https://doi.org/10.1038/ng.2607 https://www.nature.com/articles/ ng.2607\#supplementary-information. PMID: 23563609

36. Wood AR, Tyrrell J, Beaumont R, Jones SE, Tuke MA, Ruth KS, et al. Variants in the FTO and CDKAL1 loci have recessive effects on risk of obesity and type 2 diabetes, respectively. Diabetologia. 2016; 59 (6):1214-21. https://doi.org/10.1007/s00125-016-3908-5 PMID: 26961502

37. Claussnitzer M, Dankel SN, Kim H, Quon G, Meuleman W, Haugen C, et al. FTO Obesity Variant Circuitry and Adipocyte Browning in Humans. New England Journal of Medicine. 2015; 373(10):895-907. https://doi.org/10.1056/NEJMoa1502214 PMID: 26287746.

38. Graff M, Ngwa JS, Workalemahu T, Homuth G, Schipf S, Teumer A, et al. Genome-wide analysis of $\mathrm{BMI}$ in adolescents and young adults reveals additional insight into the effects of genetic loci over the life course. Human Molecular Genetics. 2013; 22(17):3597-607. https://doi.org/10.1093/hmg/ddt205 PMID: 23669352

39. Vattikuti S, Guo J, Chow CC. Heritability and Genetic Correlations Explained by Common SNPs for Metabolic Syndrome Traits. PLoS genetics. 2012; 8(3):e1002637. https://doi.org/10.1371/journal.pgen. 1002637 PMID: 22479213

40. Kim S, Yu N-K, Kaang B-K. CTCF as a multifunctional protein in genome regulation and gene expression. Experimental \&Amp; Molecular Medicine. 2015; 47:e166. https://doi.org/10.1038/emm.2015.33 PMID: 26045254

41. Chen Q, Quan C, Xie B, Chen L, Zhou S, Toth R, et al. GARNL1, a major RalGAP alpha subunit in skeletal muscle, regulates insulin-stimulated RalA activation and GLUT4 trafficking via interaction with 143-3 proteins. Cell Signal. 2014; 26(8):1636-48. Epub 2014/04/29. https://doi.org/10.1016/j.cellsig.2014. 04.012 PMID: 24768767.

42. Lin WY, Chan CC, Liu YL, Yang AC, Tsai SJ, Kuo PH. Performing different kinds of physical exercise differentially attenuates the genetic effects on obesity measures: Evidence from 18,424 Taiwan Biobank participants. PLoS genetics. 2019; 15(8):e1008277. Epub 2019/08/02. https://doi.org/10.1371/journal. pgen.1008277 PMID: 31369549; PubMed Central PMCID: PMC6675047.

43. Chen $\mathrm{CH}$, Yang JH, Chiang CWK, Hsiung CN, Wu PE, Chang LC, et al. Population structure of Han Chinese in the modern Taiwanese population based on 10,000 participants in the Taiwan Biobank project. Hum Mol Genet. 2016; 25(24):5321-31. Epub 2016/11/01. https://doi.org/10.1093/hmg/ddw346 PMID: $27798100 ;$ PubMed Central PMCID: PMC6078601.

44. Lo YH, Cheng HC, Wang HY, Peng CW, Chen CY, Lin KP, et al. Detecting genetic ancestry and adaptation in the Taiwanese Han people. Molecular biology and evolution. 2020. Epub 2020/11/11. https://doi. org/10.1093/molbev/msaa276 PMID: 33170928

45. Chang CC, Chow CC, Tellier LC, Vattikuti S, Purcell SM, Lee JJ. Second-generation PLINK: rising to the challenge of larger and richer datasets. Gigascience. 2015; 4:7. Epub 2015/02/28. https://doi.org/ 10.1186/s13742-015-0047-8 PMID: 25722852; PubMed Central PMCID: PMC4342193.

46. Delaneau $\mathrm{O}$, Marchini J, Zagury J-F. A linear complexity phasing method for thousands of genomes. Nature Methods. 2011; 9:179. https://doi.org/10.1038/nmeth.1785 https://www.nature.com/articles/ nmeth.1785\#supplementary-information. PMID: 22138821

47. Marchini J, Howie B, Myers S, McVean G, Donnelly P. A new multipoint method for genome-wide association studies by imputation of genotypes. Nature genetics. 2007; 39(7):906-13. Epub 2007/06/19. https://doi.org/10.1038/ng2088 PMID: 17572673.

48. Yang J, Lee SH, Goddard ME, Visscher PM. GCTA: a tool for genome-wide complex trait analysis. Am J Hum Genet. 2011; 88(1):76-82. Epub 2010/12/21. https://doi.org/10.1016/j.ajhg.2010.11.011 PMID: $21167468 ;$ PubMed Central PMCID: PMC3014363.

49. Bulik-Sullivan BK, Loh PR, Finucane HK, Ripke S, Yang J, Schizophrenia Working Group of the Psychiatric Genomics C, et al. LD Score regression distinguishes confounding from polygenicity in genomewide association studies. Nature genetics. 2015; 47(3):291-5. Epub 2015/02/03. https://doi.org/10. 1038/ng.3211 PMID: 25642630; PubMed Central PMCID: PMC4495769.

50. Yang J, Ferreira T, Morris AP, Medland SE, Genetic Investigation of ATC, Replication DIG, et al. Conditional and joint multiple-SNP analysis of GWAS summary statistics identifies additional variants influencing complex traits. Nature genetics. 2012; 44(4):369-75, S1-3. Epub 2012/03/20. https://doi.org/10. 1038/ng.2213 PMID: 22426310; PubMed Central PMCID: PMC3593158. 
51. Pruim RJ, Welch RP, Sanna S, Teslovich TM, Chines PS, Gliedt TP, et al. LocusZoom: regional visualization of genome-wide association scan results. Bioinformatics. 2010; 26(18):2336-7. https://doi.org/ 10.1093/bioinformatics/btq419 PMID: 20634204

52. Willer CJ, Li Y, Abecasis GR. METAL: fast and efficient meta-analysis of genomewide association scans. Bioinformatics. 2010; 26(17):2190-1. Epub 2010/07/10. https://doi.org/10.1093/bioinformatics/ btq340 PMID: 20616382; PubMed Central PMCID: PMC2922887.

53. Buniello A, MacArthur JAL, Cerezo M, Harris LW, Hayhurst J, Malangone C, et al. The NHGRI-EBI GWAS Catalog of published genome-wide association studies, targeted arrays and summary statistics 2019. Nucleic acids research. 2019; 47(D1):D1005-D12. Epub 2018/11/18. https://doi.org/10.1093/nar/ gky1120 PMID: 30445434; PubMed Central PMCID: PMC6323933.

54. Watanabe K, Taskesen E, van Bochoven A, Posthuma D. Functional mapping and annotation of genetic associations with FUMA. Nature communications. 2017; 8(1):1826. Epub 2017/12/01. https:// doi.org/10.1038/s41467-017-01261-5 PMID: 29184056; PubMed Central PMCID: PMC5705698.

55. Wang K, Li M, Hakonarson H. ANNOVAR: functional annotation of genetic variants from high-throughput sequencing data. Nucleic acids research. 2010; 38(16):e164. Epub 2010/07/06. https://doi.org/10. 1093/nar/gkq603 PMID: 20601685; PubMed Central PMCID: PMC2938201.

56. Schmitt Anthony D, Hu M, Jung I, Xu Z, Qiu Y, Tan Catherine L, et al. A Compendium of Chromatin Contact Maps Reveals Spatially Active Regions in the Human Genome. Cell Reports. 2016; 17(8):2042-59. https://doi.org/10.1016/j.celrep.2016.10.061 PMID: 27851967

57. Bulik-Sullivan B, Finucane HK, Anttila V, Gusev A, Day FR, Loh PR, et al. An atlas of genetic correlations across human diseases and traits. Nature genetics. 2015; 47(11):1236-41. Epub 2015/09/29. https://doi.org/10.1038/ng.3406 PMID: 26414676; PubMed Central PMCID: PMC4797329. 